\title{
Swirl-Nozzle Interaction Experiments: Influence of Injection-Reservoir Pressure and Injection Time
}

\author{
L. Hirschberg* \\ German Aerospace Center, 10623 Berlin, Germany \\ F. Bake ${ }^{\dagger}$ \\ German Aerospace Center, 10623 Berlin, Germany \\ K. Knobloch \\ German Aerospace Center, 10623 Berlin, Germany
}

S. J. Hulshoff ${ }^{\S}$

Delft University of Technology, 2629 HS Delft, the Netherlands

\footnotetext{
$c_{\theta}=$ sound speed in injection reservoir, $\mathrm{m} \cdot \mathrm{s}^{-1}$

$c_{\mathrm{th}}=$ sound speed at nozzle throat, $\mathrm{m} \cdot \mathrm{s}^{-1}$

$c_{1} \quad=$ sound speed in upstream section, $\mathrm{m} \cdot \mathrm{s}^{-1}$

$c_{2}=$ sound speed in downstream section, $\mathrm{m} \cdot \mathrm{s}^{-1}$

$f_{\text {ref }}=$ pistonphone reference frequency, $\mathrm{Hz}$

$L_{0} \quad=\quad$ effective injection cross-section fit parameter, $\mathrm{m}$

$L_{1} \quad=\quad$ effective injection cross-section fit parameter, $\mathrm{m}$

$M_{1} \quad=$ Mach number in the upstream section

$M_{2} \quad=$ Mach number in the downstream section

$M_{\mathrm{inj}} \quad=\quad$ effective injection-jet Mach number

$\dot{m}_{1}=$ mass flux in the upstream section, $\mathrm{kg} \cdot \mathrm{s}^{-1}$

$\dot{m}_{\theta} \quad=$ mass flux of tangential injection, $\mathrm{kg} \cdot \mathrm{s}^{-1}$

$p_{1}^{\prime} \quad=\quad$ upstream acoustic pressure signal, $\mathrm{Pa}$

$p_{2}^{\prime} \quad=$ downstream acoustic pressure signal, $\mathrm{Pa}$

$\left|p_{\text {ref }}^{\prime}\right|=$ pistonphone calibration pressure, $\mathrm{dB}$

$p_{\theta} \quad=$ absolute injection reservoir pressure, bar

$p_{\mathrm{atm}}=$ atmospheric pressure, bar

$R_{1} \quad=\quad$ upstream pipe section radius, $\mathrm{m}$

*DLR-DAAD Postdoctoral Fellow, German Aerospace Center, Institute of Propulsion Technology, Engine Acoustics, Mueller-Breslau-Straße 8, 10623 Berlin, Germany, AIAA Member.

†Team Leader Combustion Acoustics, German Aerospace Center, Institute of Propulsion Technology, Engine Acoustics, Mueller-Breslau-Straße 8, 10623 Berlin, Germany, AIAA Member.

†Combustion Acoustics Scientist, German Aerospace Center, Institute of Propulsion Technology, Engine Acoustics, Mueller-Breslau-Straße 8, 10623 Berlin, Germany, AIAA Member.

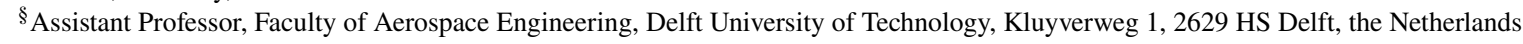


$R_{2} \quad=\quad$ downstream pipe section radius, $\mathrm{m}$

$R_{\theta} \quad=\quad$ injector outlet surface radius, $\mathrm{m}$

$R_{\text {valve }}=$ valve outlet surface radius of a single port, $\mathrm{m}$

$R_{\text {eff }} \quad=$ effective valve outlet surface radius, $\mathrm{m}$

$R_{\mathrm{th}} \quad=$ nozzle throat radius, $\mathrm{m}$

$R^{*} \quad=$ critical injection radius, $\mathrm{m}$

$S_{j} \quad=$ effective injection-jet cross-section, $\mathrm{m}^{2}$

$T_{\mathrm{atm}}=$ temperature in the laboratory, $\mathrm{K}$

$V_{\text {set }} \quad=$ volume of upstream settling chamber $\mathrm{m}^{3}$

$V_{\text {valve }}=$ valve trigger pulse, $\mathrm{V}$

$V_{\theta} \quad=$ volume of tangential injection reservoir, $\mathrm{m}^{3}$

$\alpha_{\mathrm{crt}} \quad=$ fit coefficient for fit of critical tangential-injection data, $\mathrm{kg} \cdot \mathrm{s}^{-1} \cdot \mathrm{Pa}^{-1}$

$\gamma \quad=$ specific heat ratio, $\gamma \equiv c_{p} / c_{v}$

$\rho \quad=\quad$ local density $\mathrm{kg} \cdot \mathrm{m}^{-3}$

$\rho_{1} \quad=$ density upstream from the choked convergent-divergent nozzle, $\mathrm{kg} \cdot \mathrm{m}^{-3}$

$\rho_{2} \quad=$ density downstream from the choked convergent-divergent nozzle $\mathrm{kg} \cdot \mathrm{m}^{-3}$

$\rho_{\theta} \quad=$ injection air density, $\mathrm{kg} \cdot \mathrm{m}^{-3}$

$\tau_{\theta} \quad=$ injection time, $\mathrm{s}$

\section{Introduction}

Engineering systems employing turbulent combustion usually have high levels of noise production, due both to direct and indirect combustion-noise sources. Direct sources, due to unsteady gas expansion in flames, have been widely studied [1-4]. Indirect sources include entropy noise, caused by entropy patches (localised gradients in the flow's thermodynamic state which arise from non-uniform combustion), and vorticity noise, caused by vortices. Entropy patches and vortices produce sound waves as they exit the area of combustion through a nozzle or turbine. Some of these are radiated into the environment, and some are reflected back into the combustion chamber. The latter can induce the production of new entropy and vorticity noise sources. Under certain circumstances this results in a feedback loop that promotes combustion instability. Indirect-combustion noise driven thermoacoustic combustion-chamber instability is a problem in aeroengines, electrical-power generation turbines and solid rocket motors [2, 3, 5, 7, [-12]. Entropy noise has been widely studied, as evidenced by the high number of citations of two seminal articles by Marble and Candel [13] and Ffowcs Williams and Howe [14]. Vorticity noise has received far less attention.

In the case of vorticity noise, one should distinguish between sound produced by vorticity oriented normal to the 
main flow, and that produced by vorticity oriented parallel (swirl) to the main flow. Most combustors used in gas turbines and aeroengines are swirl-stabilised, i.e, in these a significant vorticity component is created parallel to the main flow. Kings and Bake [15] investigated parallel-component vorticity noise experimentally in isolation, by means of pulsated tangential air injections into a steady flow upstream from a choked nozzle.

Recently, Hirschberg et al. [16] argued that in this experiment, sound production is due to a reduction of the mass flux through the nozzle as the upstream-generated swirling flow structure is ingested. The analysis of Hirschberg et al. [16] employed a quasi-steady quasi-cylindrical analytical model, which indicated that the upstream swirl-intensity change due to unsteady-tangential injection was the driving parameter for sound production. During ingestion the axial vortex (swirl) is elongated. Due to conservation of angular momentum the tangential velocity is increased. This implies that part of the available stagnation enthalpy is not available for the axial flow velocity component. Consequently, the mass flow through the critical nozzle throat reduces. This reduction in mass flow induces a diminished axial flow velocity downstream of the nozzle and an expansion wave is generated in the downstream pipe. Assuming quasi-steady behavior and an anechoic downstream pipe termination, this expansion wave will have a constant amplitude as long as the swirl at the nozzle throat is not changed.

A major problem in the experiment of Kings and Bake [15] is that acoustic reflections at the downstream open-pipe termination obscure the signal of the swirl-nozzle interaction. This spurious effect was mitigated in a new series of experiments presented here, through the extension of the downstream pipe. Additional improvements to the swirl-nozzle interaction experiment were identified. These included using an injection reservoir of known volume $V_{\theta}$ for the unsteady tangential injection, and the calibration of the tangential mass-flow rate. The upstream settling chamber volume $V_{\text {set }}$ was doubled. Furthermore, the pressure fluctuations upstream from the nozzle were recorded and analyzed.

Here, measurement results using the improved swirl-nozzle interaction setup are reported for the first time. The improved setup made the downstream detection and quantitative measurement of the swirl-nozzle interaction generated acoustic amplitude possible, a feat which had not previously been achieved. The influence of the duration of the tangential injection time was investigated. This provides an indication of the validity range of the quasi-steady model reported in Ref. [16].

\section{Description of experimental setups}

The improved swirl-nozzle interaction setup and its measurement procedure are described in section II.A The setup and procedure used to calibrate the tangentially injected mass-flow rate are described in section II.B.

\section{A. Acoustic measurements setup}

In Fig. 1 a sketch of the acoustic measurement setup is shown. The upstream part of the setup consisted of a settling chamber $\left(V_{\text {set }}=10.5 \times 10^{-3} \mathrm{~m}^{3}\right)$ with a bell-mouth inlet to a tube section. This $220 \mathrm{~mm}$ long tube section had a 


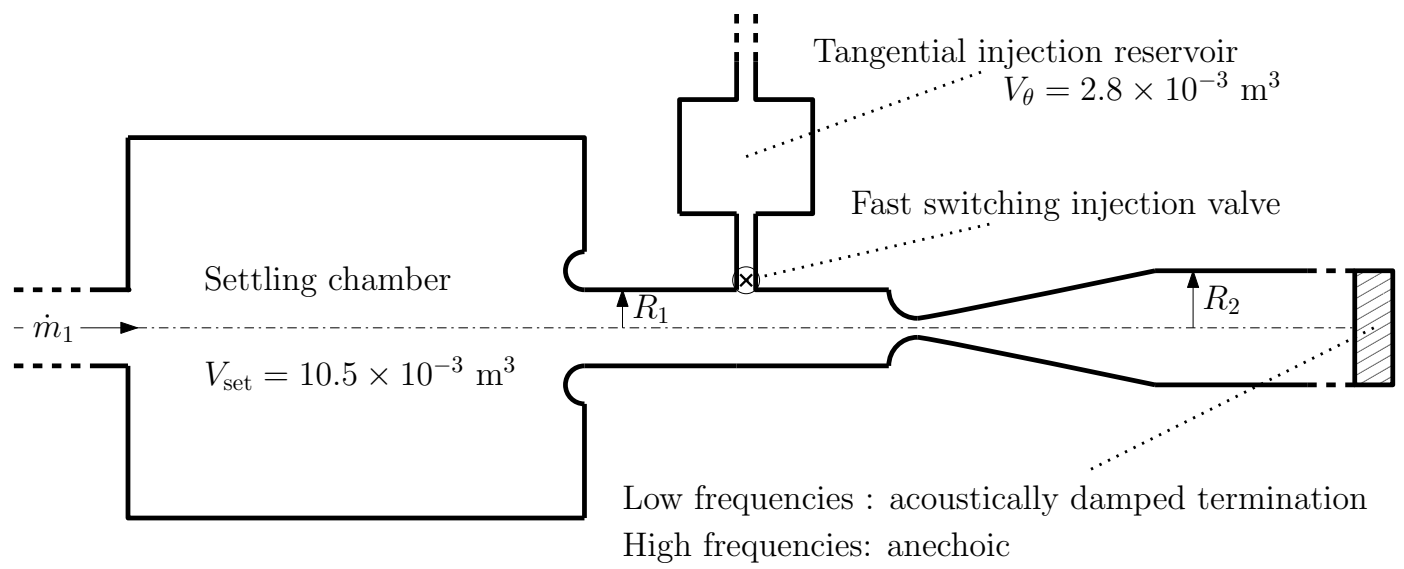

Fig. 1 Sketch of swirl-nozzle interaction setup.

$R_{1}=15 \mathrm{~mm}$ radius. A single tangential-injection port module (Fig. 2) was connected to the downstream end of the aforementioned tube section. The injection port module was composed of a $70 \mathrm{~mm}$ long upstream pipe section with a radius of $R_{1}=15 \mathrm{~mm}$. Tangential injection of air into the stationary axial base flow was performed through a port machined in the middle of the module, which had a small nozzle of outlet diameter $R_{\theta}=1.25 \mathrm{~mm}$. Air injection was done using a fast-switching valve for a variable duration of $\tau_{\theta}$. This was repeated 300 times every $3 \mathrm{~s}$. The valve was connected to the injection port through a $37 \mathrm{~mm}$ long tube with a $4 \mathrm{~mm}$ inner diameter. The nominal opening and closing times of the valve were reported by the manufacturer to be $2.5 \mathrm{~ms}$ [17]. Although the valve has an opening time of $2.5 \mathrm{~ms}$, the authors found that the pressure response measured, by means of a GRAS 40BP 1/4" ext. polarized pressure microphone mounted flush in the wall $185 \mathrm{~mm}$ upstream from the tangential-injection point, shows that the valve takes longer to close. This will be elaborated on in section III.B The valve had three injection holes each with radius $R_{\text {valve }}=0.90 \mathrm{~mm}$. The effective radius of the injection valve had an estimated maximum effective opening radius $R_{\text {eff }}=\sqrt{3}\left(R_{\text {valve }}\right) \simeq 1.56 \mathrm{~mm}$. Analysis of measurements, section III.A. will show the effective radius of the choked valve to be significantly smaller. The injection valve was connected to a $V_{\theta}=2.8 \times 10^{-3} \mathrm{~m}^{3}$ injection reservoir, by means of a $150 \mathrm{~mm}$ long plastic hose with an inner diameter of $12 \mathrm{~mm}$. The injection reservoir was put under a pressure $p_{\theta}$, by means of a compressed-air supply system, connected to the reservoir through a $3.5 \mathrm{~m}$ long $12 \mathrm{~mm}$ inner diameter hose. $p_{\theta}$ was set by means of a valve with a mechanical dial. The dial values were calibrated a posteriori using a NetScanner ${ }^{\mathrm{TM}}$ System Model 9116 manometer. Design details about the injection valve can be found in Ref. [17], and about how it was operated in Ref. [15].

The tangential injection module was followed downstream by a $50 \mathrm{~mm}$ long uniform tube of radius $R_{1}=15 \mathrm{~mm}$. This tube was connected to a converging-diverging nozzle with throat radius $R_{\mathrm{th}}=3.25 \mathrm{~mm}$ (cross-sectional surface contraction ratio 1/16). Hence, the distance between the tangential-injection port and the nozzle inlet was $85 \mathrm{~mm}$. Downstream from the conical divergent part of nozzle $(250 \mathrm{~mm}$ long) was a uniform tube with a radius of $20 \mathrm{~mm}$ and a 


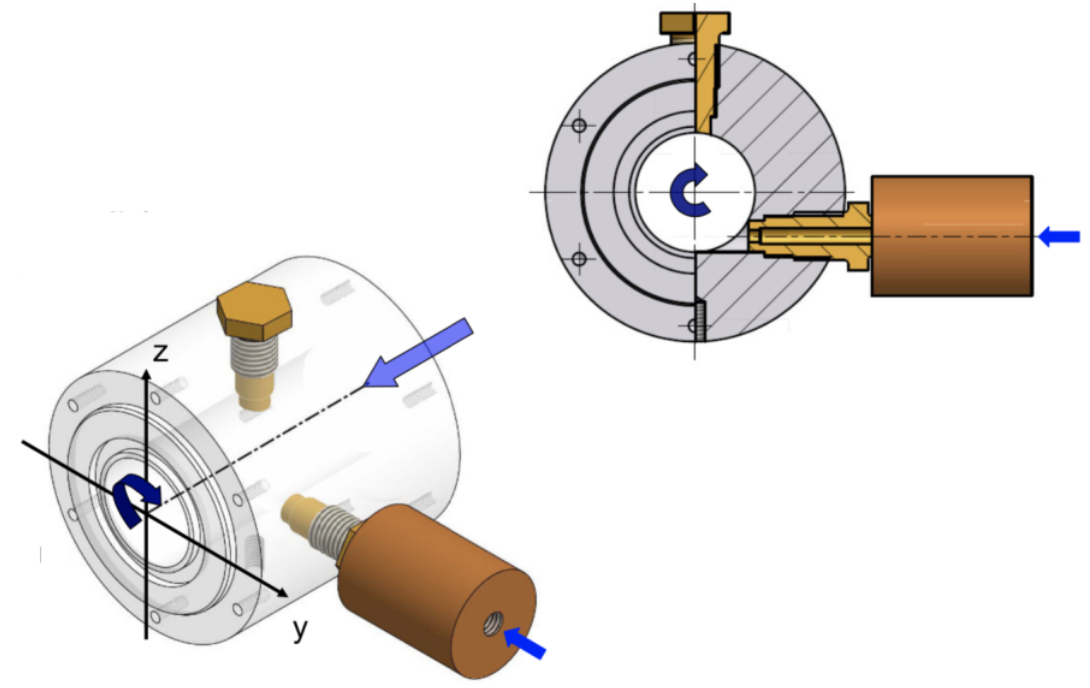

Fig. 2 The tangential-injection module.

length of $1020 \mathrm{~mm}$ referred to as the "microphone section."

A GRAS 40BP 1/4" ext. polarized pressure microphone was mounted flush in its walls, calibrated using a Brüel \& Kjaer model 4228 pistonphone at $\left|p_{\text {ref }}^{\prime}\right|=123.92 \mathrm{~dB}$ and $f_{\text {ref }}=251.2 \mathrm{~Hz}$, at a distance $1150 \mathrm{~mm}$ from the nozzle throat. It was used to detect pressure waves generated by swirl-nozzle interaction. This acoustic signal $p_{2}^{\prime}$ was recorded using an OROS OR-36 12-channel analyzer with NVGate data acquisition system software, and sampling frequency $f_{s}=16384 \mathrm{~Hz}$

An acoustically damping termination was connected to the microphone section by a flexible tube (radius $R_{2}=20 \mathrm{~mm}$ and $4000 \mathrm{~mm}$ length). The effect of acoustic reflections from this termination at low frequencies is discussed in Ref. [16]. It is only anechoic for high frequencies (above $300 \mathrm{~Hz}$ ). The flexible tube was approximately four times longer than the one used by Kings and Bake [15]. This is a significant improvement, as it prolongs the back-and-forth travel time of an acoustic wave from the microphone to the downstream termination by more than a factor two. This enables the measurement of the acoustic amplitude due to swirl-nozzle interaction in the downstream mic section, without the influence of any acoustic reflections for a period of ca. $30 \mathrm{~ms}$. Note that the observation time is also restricted by the limited low frequency response of the microphone to about $40 \mathrm{~ms}$.

A stationary non-swirling axial base flow was created by imposing a mass-flow rate of $\dot{m}_{1}=43 \mathrm{~kg} \cdot \mathrm{h}^{-1}$ in the settling chamber. This was done using a Bronckhorst F-203AV linear resistance flow controller. At this mass-flow rate, choked nozzle conditions were obtained with a reservoir pressure $p_{1}=1.12$ bar. This imposed an upstream nominal nozzle inlet Mach number of $M_{1}=3.67 \times 10^{-2}$. The pressure $p_{2}$ in the section downstream from the nozzle was atmospheric $p_{\text {atm }}=1.01$ bar with a Mach number of $M_{2}=2.27 \times 10^{-2}$. 


\section{B. Tangential mass-flow rate measurement setup}

The tangential injection mass-flow rate $\dot{m}_{\theta}$ was determined from the measured tangential injection reservoir pressure $p_{\theta}$. To determine $\dot{m}_{\theta}$ as a function of the injection reservoir pressure $p_{\theta}$, steady flow measurements were used. For this purpose, a Bronckhorst F-203AV linear resistance flow meter was installed upstream of the tangential injection reservoir. The axial injection $\dot{m}_{1}$ was set to zero. I.e., during the calibration procedure $p_{1}=p_{2}=p_{\text {atm }}$. This was done to ensure that a $60 \mathrm{~s}$ tangential injection would not cause a dangerous increase of pressure in the setup (Fig. 1). A $60 \mathrm{~s}$ tangential injection time was necessary to ensure that the Bronckhorst flow meter measured $\dot{m}_{\theta}$ reliably. $p_{\theta}$ was measured using a NetScanner ${ }^{\mathrm{TM}}$ System Model 9116 manometer, after $55 \mathrm{~s}$ of tangential injection. A tangential injection outlet port radius $R_{\theta}=1.25 \mathrm{~mm}$ was used. The temperature in the lab was measured, and found to be $T_{\mathrm{atm}}=293.9 \mathrm{~K}$.

\section{Results}

\section{A. Tangential mass-flow rate measurement results}

Two tangential-injection regimes were identified, one for which the injector was choked and the other not. In the following these are referred to as critical and subcritical tangential injection, respectively.

In Fig. 3, the tangential injection mass-flow rate $\dot{m}_{\theta}$ is plotted as a function of $p_{\theta} / p_{1}$, in which the two regimes are visible. The first (open circles) obtained with subcritical tangential injection $\left(p_{\theta} / p_{1}<2.0\right)$, and the second (crosses) obtained with critical tangential injection $\left(p_{\theta} / p_{1}>2.5\right)$.

The data was fitted using the least squares matlab tool "polyfit." This yielded, for the critical data:

$$
\left(\dot{m}_{\theta}\right)_{\mathrm{crt}, \mathrm{fit}}=\alpha_{\mathrm{crt}} p_{\theta}
$$

where $\alpha_{\mathrm{crt}}=6.676 \times 10^{-9} \mathrm{~kg} \cdot \mathrm{s}^{-1} \cdot \mathrm{Pa}^{-1}$.

The critical injection radius $R^{*}$ of the tangential-injection valve, using

$$
R^{*}=\sqrt{\frac{p_{\mathrm{atm}} \dot{m}_{\theta}}{\pi c_{\theta} p_{\theta} \rho_{\mathrm{atm}}}}\left(\frac{\gamma+1}{2}\right)^{(\gamma+1) /(4(\gamma-1))}
$$

where $\gamma=1.4$ is the constant pressure to constant volume heat capacity ratio for dry air, $c_{\theta}=344 \mathrm{~m} \cdot \mathrm{s}^{-1}$ the sound speed in the injection reservoir, and $\rho_{\mathrm{atm}}=1.20 \mathrm{~kg} \cdot \mathrm{m}^{-3}$ the density of the atmospheric air. Eq. (1), can be substituted into Eq. (2) to determine $R^{*}$. Doing so, one finds $R^{*}=0.948 \mathrm{~mm}$

One notes that the geometrical effective radius $R_{\mathrm{eff}}=1.55 \mathrm{~mm}$ of the valve, and the tangential-injection port radius $R_{\theta}=1.25 \mathrm{~mm}$ are larger than $R^{*}$. This means that for critical flow, $\dot{m}_{\theta}$ is fixed by the valve. Thus, when performing acoustic measurements, the tangential mass-flow rate $\dot{m}_{\theta}$ can be calculated using 


$$
\dot{m}_{\theta}=\pi\left(R^{*}\right)^{2} \rho_{1} c_{\theta} \frac{p_{\theta}}{p_{1}}\left(\frac{2}{\gamma+1}\right)^{(\gamma+1) /(2(\gamma-1))} .
$$

were $\rho_{1}$ and $p_{1}$ are the density and absolute pressure upstream from the convergent-divergent choked nozzle.

For the subcritical tangential-injection data $p_{\theta} / p_{1}<2.0$, the following fit is proposed

$$
\dot{m}_{\theta}=\rho_{1} c_{1}\left(\frac{p_{\theta}}{p_{1}}\right)^{(\gamma-1) /(2 \gamma)} M_{\text {inj }} S_{j}
$$

where $S_{j}$ and $M_{\mathrm{inj}}$ are the effective injection jet cross-section and injection Mach number, respectively. $S_{j}$ is calculated using the following fit relation

$$
S_{j}=\pi\left(L_{1} M_{\mathrm{inj}}+L_{0}\right)^{2}
$$

where $L_{0}=0.79313 \mathrm{~mm}$ and $L_{1}=0.13027 \mathrm{~mm}$, and the effective jet Mach number $M_{\text {inj }}$ is determined using

$$
M_{\text {inj }}=\sqrt{\frac{2}{\gamma-1}\left(\left(\frac{p_{\theta}}{p_{1}}\right)^{(\gamma-1) / \gamma}-1\right)} .
$$

Eq. (4) is based on a compressible isentropic flow model between the valve and the injection jet. Additional losses occur downstream from the valve, and upstream of the injection port. Therefore, the effective injection Mach number $M_{\text {inj }}$ is not an actual jet Mach number and can be larger than unity.

\section{B. Acoustic measurement results}

The acoustic signals, recorded as a result of the 300 consecutive $3 \mathrm{~s}$ measurements, were phase averaged using the technique reported in Ref. [15]. The phase-averaged signal contained strong acoustic oscillations due to e.g. a quarter-wavelength oscillation (ca. $250 \mathrm{~Hz}$ ) of the upstream pipe section of setup [16]. These spurious oscillations can clearly be seen in the non-filtered acoustic signals (finely dashed black lines) in Fig. 4

The results shown in Fig. 4 were obtained with a $10 \mathrm{~ms}$ long square pulse of $10 \mathrm{~V}$ amplitude (blue line and vertical axis on the right hand side), used to trigger the opening and closing of the valve, and absolute injection-reservoir pressure $p_{\theta}=5.09$ bar. The vertical axes, in Figs. 4(a) and 4(b) correspond to the downstream $p_{2}^{\prime}$ (microphone positioned $1150 \mathrm{~mm}$ downstream from the nozzle throat) and upstream $p_{1}^{\prime}$ (microphone positioned $270 \mathrm{~mm}$ upstream from the nozzle inlet) acoustic signals. In both, the finely dotted lines correspond to the phase-averaged signals, while the solid black line corresponds to these signals filtered using a moving-average filter with a high-pass cut-off frequency of $f_{c}=234.06 \mathrm{~Hz}$. Note that the moving-average filter causes a $2 \mathrm{~ms}$ delay of the signal. The solid vertical lines at $t=2.5 \mathrm{~ms}$ correspond to the point in time when the tangential-injection valve was opened. The vertical dashed lines at $8 \mathrm{~ms}$ correspond to the approximate moment the upstream generated swirl structure entered the nozzle. At this point the 


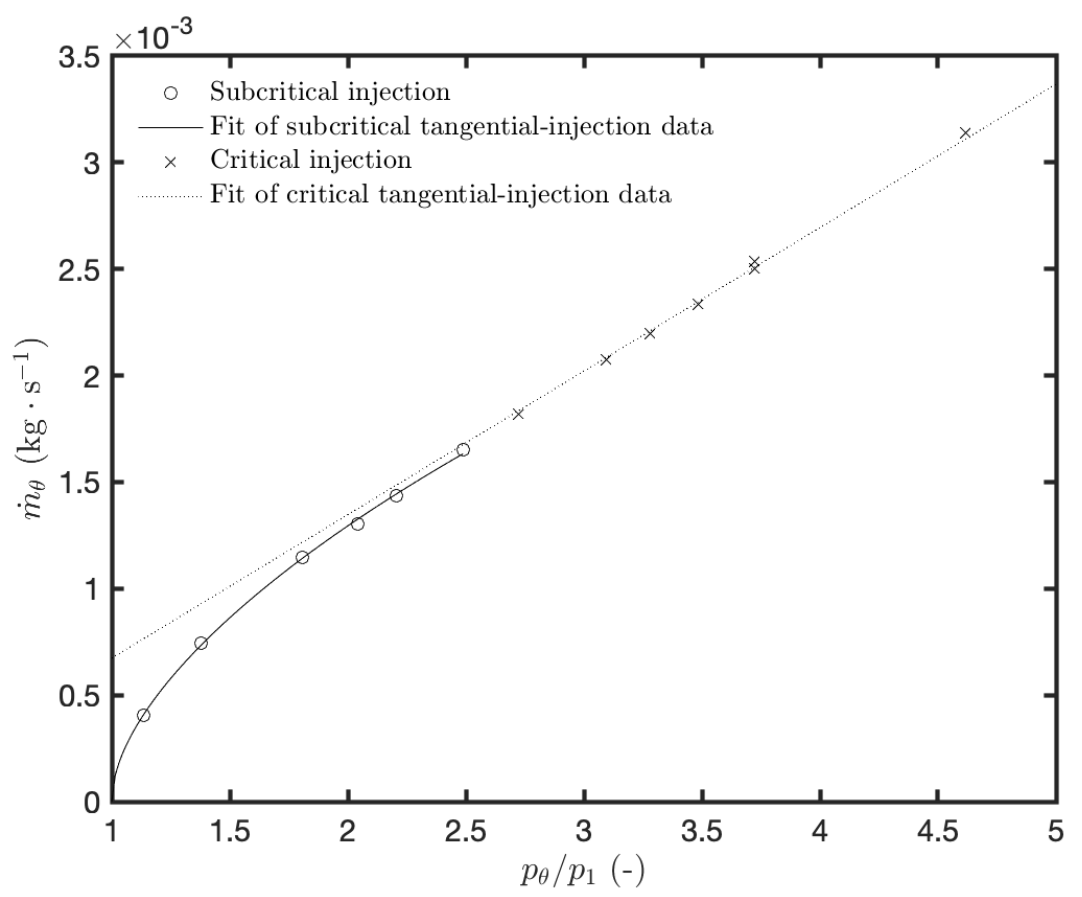

Fig. $3 \dot{m}_{\theta}$ as a function of $p_{\theta} / p_{1}$.

presence of swirl decreased the mass-flow rate through the choked nozzle, which caused an acoustic signal to be emitted downstream. This swirl-nozzle interaction signal had an amplitude of ca. $\left|p_{2}^{\prime}\right|_{\max }=140 \mathrm{~Pa}$, as can clearly be seen in Fig. 4(a). The vertical dashed-dotted lines at $27 \mathrm{~ms}$ correspond to the point in time when the valve was closed. One notes that this corresponds to the point in time were the upstream reservoir pressure $p_{1}$ ceased to rise, as can be seen in Fig. 4(b), The dotted lines at $32.5 \mathrm{~ms}$ correspond to the approximate moment at which the swirl exits the nozzle. Without the presence of swirl in the nozzle throat the mass-flow rate returns back to its swirl-free steady state value, causing $p_{2}^{\prime}$ to increase back to zero. Note that the time it takes for an plane acoustic wave to travel from the downstream microphone to the downstream termination and back is ca. $30 \mathrm{~ms}$. Thus, the $p_{2}^{\prime}$ signal for $t>37 \mathrm{~ms}$ (thick vertical line in Fig. 4(a) is polluted by a upstream traveling reflection of the signal detected at $t \simeq 7.0 \mathrm{~ms}$. Approximately $3 \mathrm{~ms}$ later, the upstream traveling reflection of signal, detected for $t<37 \mathrm{~ms}$, reaches the choked nozzle and reflects. This established a damped quarter-wavelength oscillation in the downstream section of the setup. Thus, in the presently reported measurements, the most pertinent signal solely due to swirl-nozzle interaction was detected between $t \simeq 7 \mathrm{~ms}$ and $t \simeq 37 \mathrm{~ms}$.

In the following, a simple model for the increase in upstream reservoir pressure $p_{1}$ is constructed. This done by means of an integral mass balance. An adiabatic compression and a uniform pressure in the upstream reservoir are assumed. I.e., neglecting the changes in reservoir temperature and mass flux through the nozzle, one finds 


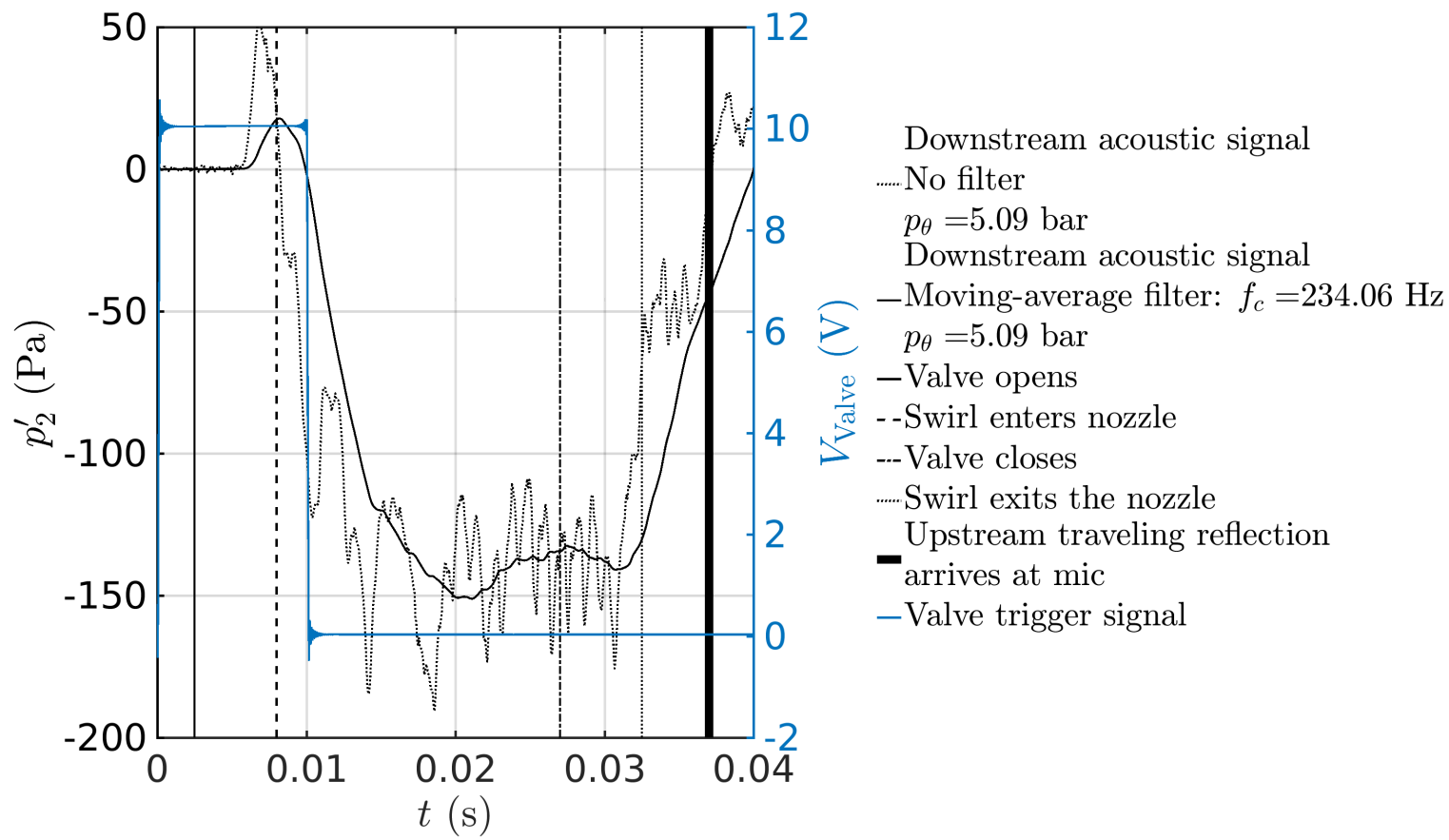

(a) Downstream microphone.

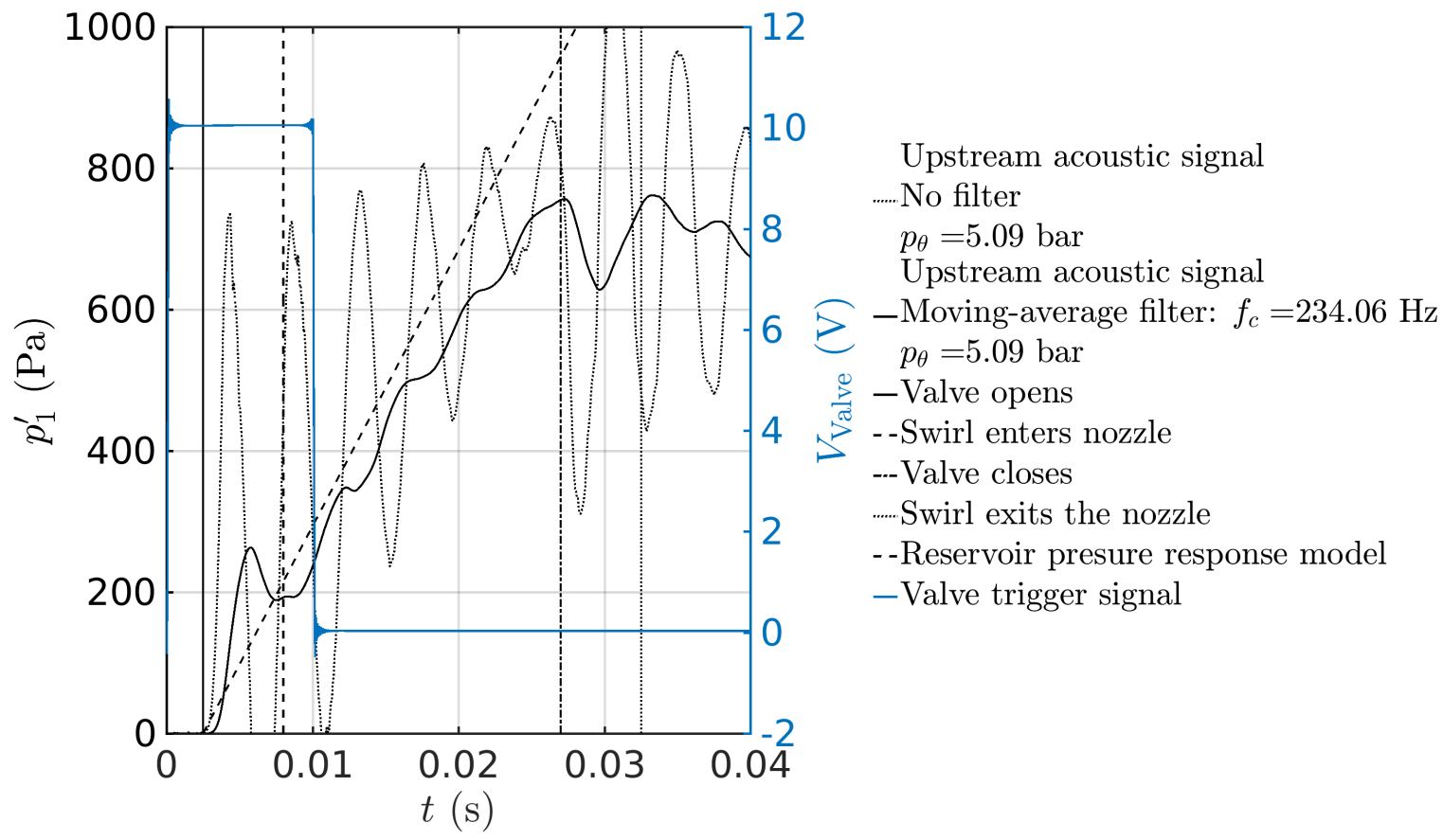

(b) Upstream microphone.

Fig. 4 (a) Downstream recorded swirl-nozzle interaction signal $p_{2}^{\prime}$. (b) Upstream recorded reservoir pressure change $p_{1}^{\prime}$ due to tangential injection. 


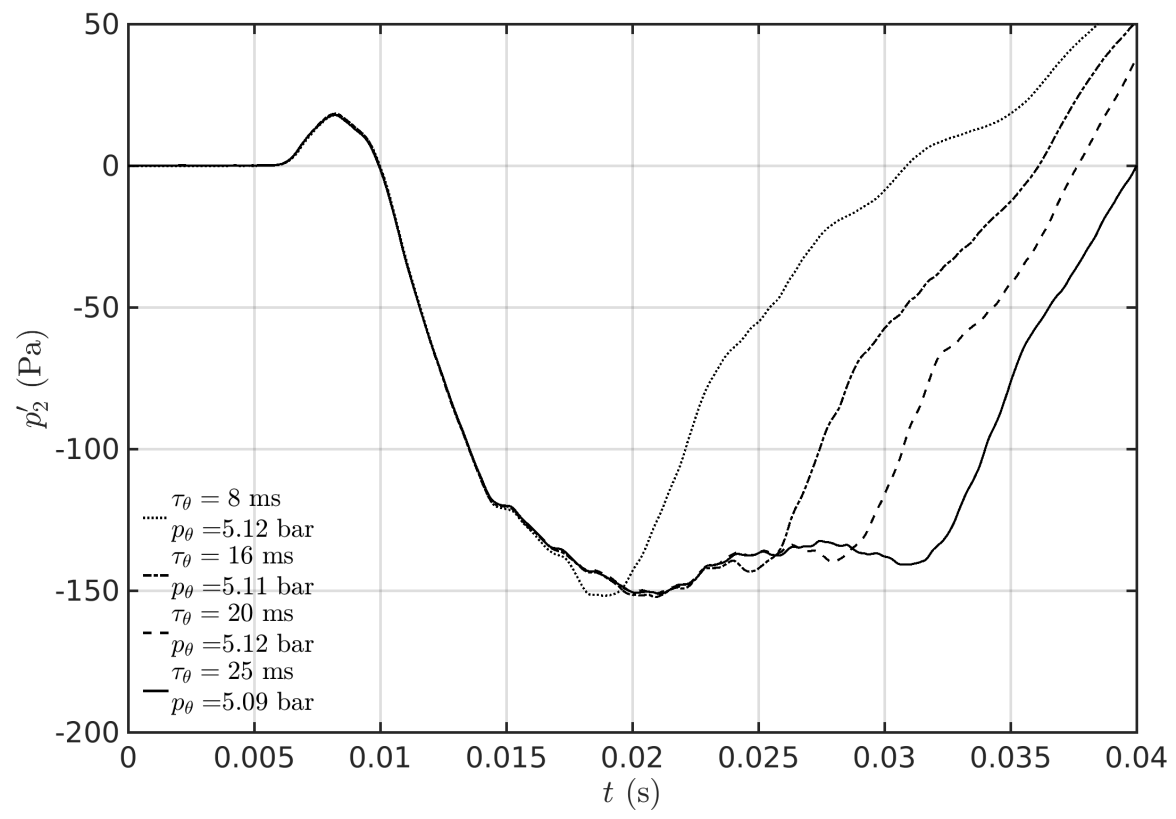

Fig. 5 Moving-average filtered $\left(f_{c}=234.06 \mathrm{~Hz}\right)$ downstream measured acoustic response $p_{2}^{\prime}$ due to swirl-nozzle interaction, for four tangential injection times $\tau_{\theta}$.

$$
\frac{V_{\text {set }}}{c_{1}^{2}} \frac{\mathrm{d} p_{1}^{\prime}}{\mathrm{d} t}=\dot{m}_{\theta}
$$

$\dot{m}_{\theta}$ is assumed constant and to corresponds to the static calibration for given constant pressure $p_{\theta}$ in the tangential injection reservoir. Using this calibration, described in section II.B. one finds $\dot{m}_{\theta}=12.5 \mathrm{~kg} \cdot \mathrm{h}^{-1}$ for $p_{\theta}=5.09$ bar. Solving for $\mathrm{d} p_{1}^{\prime} / \mathrm{d} t$, and integrating with respect to time, one finds

$$
p_{1}^{\prime}=\frac{\mathrm{d} p_{1}^{\prime}}{\mathrm{d} t}\left(t-t_{\text {open }}\right) \simeq \frac{c_{1}^{2} \dot{m}_{\theta}}{V_{\text {set }}}\left(t-t_{\text {open }}\right)
$$

were $t_{\mathrm{open}}=2.5 \mathrm{~ms}$ is the moment the tangential-injection valve is opened. The predicted linear increase in pressure is shown as a dashed black line in Fig. 4(b) One observes that Eq. (8) is fairly accurate in prediction of the initial linear increase in upstream reservoir pressure due to tangential air injection. This underpins the validity of the static calibration, described in section II.В Just before the closing of the valve, one observes a deviation of the upstream pressure from the predicted linear increase. The authors assume that this to be due to the limited low-frequency response of the microphone.

The upstream pressure fluctuations $p_{1}^{\prime}$ are partially transmitted to the downstream microphone as so-called direct sound [15, 16]. This causes the initial positive pressure pulse observed in Fig. 4(a) around $t=7 \mathrm{~ms}$.

The effect of the gradual increase of $p_{1}$ is less clear. For high upstream unsteady injection mass-flow rates it appears 


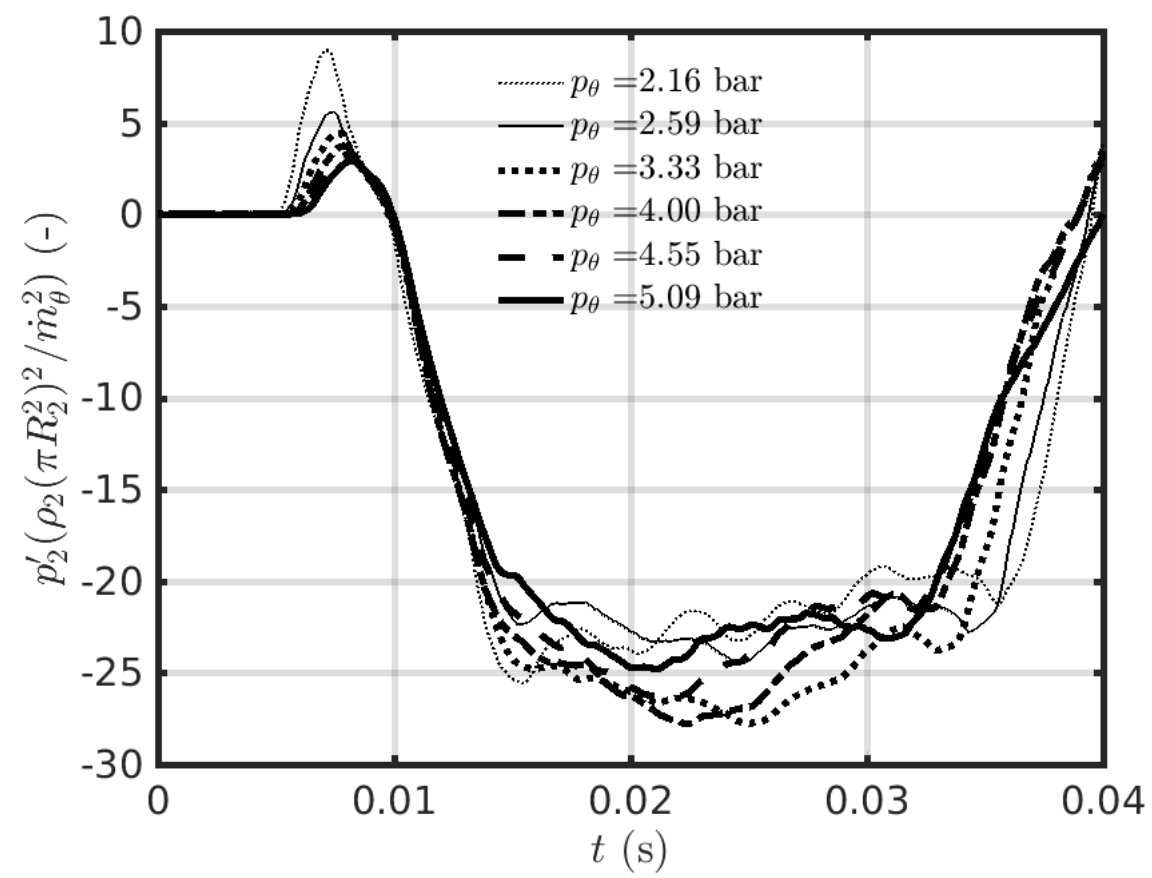

Fig. 6 Dimensionless downstream amplitude of downstream measured acoustic response $p_{2}^{\prime} \rho_{2} \pi^{2} R_{2}^{4} / \dot{m}_{\theta}^{2}$ (moving-average filtered value) due to the ingestion of swirl $\left(\tau_{\theta} \simeq 25 \mathrm{~ms}\right)$ as a function of time $t$ after the electrical trigger used to open the valve was initiated.

to be negligible. This was confirmed by experiments in which the upstream air injection was radial. However, as will be touched on later, the gradual increase of $p_{1}$ has, relatively speaking, a more significant effect on $p_{2}^{\prime}$ for lower unsteady mass-flow rates.

The results in Fig. 4(a) show that shortly after the start of the decrease in downstream pressure $p_{2}^{\prime}$, a plateau is reached at ca. $-140 \mathrm{~Pa}$. This plateau indicates quasi-steady behavior. In Fig. 5, results are shown which obtained with electrical pulse widths of $3.0 \mathrm{~ms}, 5.0 \mathrm{~ms}, 7.0 \mathrm{~ms}$, and $10 \mathrm{~ms}$. These pulse widths corresponds to $\tau_{\theta} \simeq 8 \mathrm{~ms}$ (the shortest experimentally achievable; dotted line), $\tau_{\theta} \simeq 16 \mathrm{~ms}$ (dashed-dotted line), $\tau_{\theta} \simeq 20 \mathrm{~ms}$ (dashed line), and $\tau_{\theta} \simeq 25 \mathrm{~ms}$ (solid line), respectively. This indicates that for the presently reported experiments, quasi-steady modelling remains applicable. Even for $\tau_{\theta} \simeq 8 \mathrm{~ms}$, which generates the shortest achievable swirl structure axial length (ca. $\left.6 R_{1}\right)$.

By means of the variation of $p_{\theta}$, the influence of $\dot{m}_{\theta}$ on swirl-nozzle interaction generated sound was investigated. In Fig. 6, the dimensionless (moving-average filtered) downstream measured acoustic response $p_{2}^{\prime} \rho_{2} \pi^{2} R_{2}^{4} / \dot{m}_{\theta}^{2}$ is shown as a function of time $t$. One clearly sees that there is a global collapse of the data. This means that the downstream measured acoustic response due to swirl-nozzle interaction is proportional to $\dot{m}_{\theta}^{2}$.

Note that the initial positive direct-sound pulse around $t=7 \mathrm{~ms}$ does not collapse in this dimensionless representation. This is because this pulse has an amplitude proportional to $\dot{m}_{\theta}$, as opposed to $\dot{m}_{\theta}^{2}$.

On a longer time scale, the upstream reservoir pressure $p_{1}$ gradually increases (see Fig. 4(b)). This causes a slight 
and gradual increase of $p_{2}$, which is most pronounced in this dimensionless representation for low $\dot{m}_{\theta}$. This explains why, for $p_{\theta}=2.16$ bar in Fig. 6after the sudden pressure decrease due to swirl-nozzle interaction $10 \mathrm{~ms}<t<15 \mathrm{~ms}$, one observes a gradual increase of $p_{2}^{\prime} \rho_{2} \pi^{2} R_{2}^{4} / \dot{m}_{\theta}^{2}$, while for higher $\dot{m}_{\theta}$ the pressure $p_{2}^{\prime} \rho_{2} \pi^{2} R_{2}^{4} / \dot{m}_{\theta}^{2}$ remains almost constant until the effect of the injection valve closure is observed. One notes that the valve closure time is $p_{\theta}$ dependent. This is contrasted by the valve opening time, which is apparently not influenced by $p_{\theta}$. The perfect collapse of the data during the opening phase of the valve indicates that quasi-steady behavior is likely to prevail for time scales of the order of $1 \mathrm{~ms}$. This corresponds to upstream swirl structures with an axial length of the order of $R_{1}$. In view of the contraction ratio of $\left(R_{1} / R_{\mathrm{th}}\right)^{2}=16$ of the nozzle, the axial swirl length while passing through the throat would be of the order of $16 R_{1}$. Hence, quasi-steady behavior seems a reasonable assumption.

Assuming that the upstream swirl intensity $S_{1}$ is proportional to $\dot{m}_{\theta}$, one finds that $p_{2}^{\prime} \propto S_{1}^{2}$ in the present experiments. This scaling with $S_{1}^{2}$ was predicted by the quasi-steady theory reported in Ref. [16].

\section{Conclusion}

Analysis of original swirl-nozzle interaction experiments show that the acoustic response depends quadratically on the tangential mass-flow rate $\dot{m}_{\theta}$. This was, assuming that the upstream generated swirl intensity is proportional to $\dot{m}_{\theta}$, predicted by a previously-published quasi-steady model for the swirl-nozzle interaction sound source. The tangential-injection time $\tau_{\theta}$ was varied, to investigate its influence on the acoustic response. The data shows that $\tau_{\theta}$ does not influence the amplitude of the acoustic response. This indicates that quasi-steady modelling remains applicable, even for the smallest generated swirl structure with an axial length of ca. $6 R_{1}$. This axial length is still long compared to the nozzle length. Given that structures entering a choked nozzle are elongated significantly in the axial direction, the authors infer that quasi-steady theory remains valid even for much shorter structures, with an axial length of ca. $R_{1}$. The excellent scaling of the data on $\dot{m}_{\theta}^{2}$ during the swirl-ingestion phase supports the inferred extended validity of the quasi-steady theory.

\section{Acknowledgements}

This work was carried out while Lionel Hirschberg was the beneficiary of a Deutsches Zentrum für Luft- und Raumfahrt (DLR) - Deutscher Akademischer Austauschdienst (DAAD) postdoctoral fellowship (no. 57424730). The authors thank the DLR's technical staff members Angelo Rudolphi, Sebastian Kruck, Oliver Klose, Nico Seiffert, and Lech Modrzejewski for their support.

\section{References}

[1] Strahle, W. C., “On combustion generated noise,” Journal of Fluid Mechanics, vol. 49, No. 2, 1971, pp. $399-414$. doi: $10.1017 / \mathrm{S} 0022112071002167$ 
This is the authors' preprint version of a AIAA Journal manuscript accepted for publication on April 13th 2021. $\odot$ 2021. This manuscript version is made available under the CC-BY-NC-ND 4.0 license

[2] Morgans, A. S., and Duran, I., "Entropy Noise: A Review of Theory, Progress and Challenges," Int. J. of Spray and Combustion Dynamics, Vol. 8, No. 4, 2016, pp. 285-298.

doi: $10.1177 / 1756827716651791$

[3] Dowling, A. P., and Mahmoudi, Y., "Combustion Noise," Proceedings of the Combustion Institute, Vol. 35, No. 1, 2015, pp. 65-100.

doi: $10.1016 /$ j.proci.2014.08.016

[4] Ihme, M., "Combustion and Engine-Core Noise” Annu. Rev. Fluid Mech., Vol. 49, January 2017, pp. 277-310. doi: 10.1146/annurev-fluid-122414-034542

[5] Dotson, K. W., Koshigoe, S. and Pace, K. K., "Vortex Shedding in a Large Solid Rocket Motor Without Inhibitors at the Segmented Interfaces,” Journal of Propulsion and Power, Vol. 13, No. 2, 1997, pp. 197-206.

doi: $10.2514 / 2.5170$

[6] Anthoine, J., "Experimental and Numerical Study of Aeroacoustic Phenomena in Large Solid Propellant Boosters, with application to the Ariane 5 solid rocket motor,” Ph. D. thesis, Université Libre de Bruxelles, Belgium, 2000.

[7] Hulshoff, S. J., Hirschberg, A., and Hofmans, G. C. J., "Sound production of vortex nozzle interactions", Journal of Fluid Mechanics, Vol. 439, July 2001, pp. 335-352.

doi: $10.1017 / \mathrm{S} 0022112001004554$

[8] Anthoine J., Buchlin, J.-M., and Hirschberg A., "Effect of Nozzle Cavity on Resonance in Large SRM: Theoretical Modeling", Journal of Propulsion and Power, Vol. 18, No. 2, 2002, pp. 304-311.

doi: $10.2514 / 2.5935$

[9] Hirschberg, L., Schuller, T., Collinet, J., Schram, C. , and Hirschberg, A., "Analytical model for the prediction of pulsations in a cold-gas scale-model of a Solid Rocket Motor," Journal of Sound and Vibration, Vol. 19, April 2018, pp. 445-368. doi: $10.1016 /$ j.jsv.2018.01.025

[10] Hirschberg L., Hulshoff S. J., Collinet J., Schram C., and Schuller T., "Vortex nozzle interaction in solid rocket motors: A scaling law for upstream acoustic response", The Journal of the Acoustical Society of America, Vol. 144, No. 1, 2018, pp. EL46-EL51.

doi: $10.1121 / 1.5046441$

[11] Hirschberg,L., Hulshoff, S. J., Collinet, J., Schram, C., and Schuller,T., "Influence of Nozzle Cavity on Indirect Vortex- and Entropy-Sound Production,” AIAA Journal, Vol. 57, No. 7, March 2019, pp. 3100-3103.

doi: $10.2514 / 1 . J 058138$

[12] Hirschberg, L., and Hulshoff, S. J., "Lumped-Element Model for Vortex-Nozzle Interaction in Solid Rocket Motors," AIAA Journal, Vol. 58, No. 7, July 2020 pp. 3241-3244.

doi: $10.2514 / 1 . J 058673$

13

This is the authors' preprint version of a AIAA Journal manuscript accepted for publication on April 13th 2021. @ 2021. This manuscript version is made available under the CC-BY-NC-ND 4.0 license. 
[13] Marble, F. E., and Candel, S. M., "Acoustic disturbance from gas non-uniformities convected through a nozzle," J. Sound Vib., Vol. 55, 1977, pp. 225-243.

doi: $10.1016 / 0022-460 X(77) 90596-X$

[14] Ffowcs Williams, J. E., and Howe, M. S., "The generation of sound by density inhomogeneities in low Mach number nozzle flows," Journal of Fluid Mechanics, vol. 70, no. 3, 1975, pp. 605-622.

doi: $10.1017 / \mathrm{S} 0022112075002224$

[15] Kings, N., and Bake, F., "Indirect combustion noise: noise generation by accelerated vorticity in a nozzle flow," International Journal of Spray and Combustion Dynamics, Vol. 2, No. 3, 2010, pp. 253-266.

doi: $10.1260 / 1756-8277.2 .3 .253$

[16] Hirschberg, L., Hulshoff, S. J., and Bake, F., "Sound Production due to Swirl-Nozzle Interaction: Model-Based Analysis of Experiments," AIAA Journal, Published online on Nov. 11th 2020 doi: 10.2514/1.J059669

[17] Neuhaus, D., and Röhle, I., “Schnellschaltende Ventile für Anwendungen in der Luft und Raumfahrt," Deutscher Luft- und Raumfahrtkongress, November 2006. 